Proceedings of the 5th Workshop on Quantum Chaos and Localisation Phenomena, Warsaw, Poland, May 20-22, 2011

\title{
Inverse Problems for Quantum Graphs: Recent Developments and Perspectives
}

\author{
P. KuRASOV ${ }^{a, b, c, *}$ \\ ${ }^{a}$ Dept. of Mathematics, LTH, Lund Univ., Box 118, 22100 Lund, Sweden \\ ${ }^{b}$ Dept. of Mathematics, Stockholm Univ., 106 91, Stockholm, Sweden \\ ${ }^{c}$ Dept. of Physics, St. Petersburg Univ., 198904 St. Peterhof, Russia
}

\begin{abstract}
An introduction into the area of inverse problems for the Schrödinger operators on metric graphs is given. The case of metric finite trees is treated in detail with the focus on matching conditions. For graphs with loops we show that for almost all matching conditions the potential on the loop is not determined uniquely by the Titchmarsh-Weyl function. The class of all admissible potentials is characterized.
\end{abstract}

PACS: 03.65.Nk, 73.63.-b, 85.35.-p

\section{Introduction}

In this paper we are going to describe recent status of the inverse problem for compact quantum graphs - the Schrödinger operators on metric graphs when the spectral data consist of the Titchmarsh-Weyl (TW-matrix, TW-function, see rigorous definition in Sect. 2) matrix function. From our point of view it is natural to use these spectral data, since it is the TW-function which can be measured in boundary experiments. It can also be calculated from the scattering matrix for the graph obtained by attaching infinite leads to the original compact graph. Avdonin, Belishev, Brown, Vakulenko, and Weikard $[1,2,5-7,10]$ have contributed into the development of this approach.

Other sets of spectral data for quantum graphs have been considered. For example Yurko generalized the spectral mapping approach [37-43]. The corresponding spectral data consist of the spectra of the Schrödinger operators with different matching conditions at certain internal vertices including the Dirichlet conditions which correspond to disjoint edges. Obtaining of these data in practice requires cutting of one or several internal vertices, while our approach based on TW-function can be considered as an attempt to solve the inverse problem using boundary observations.

These two approaches can be seen as natural continuations of the classical methods developed to solve the inverse problem on one interval. In these methods the inverse problem is solved using the Dirichlet-Dirichlet and Dirichlet-Neumann spectra (developed by LevitanGasymov and McKean-Trubowitz [13, 14, 28, 30], corresponds to Yurko's approach) and the TitchmarshWeyl function (developed by Faddeev-Gelfand-LevitanMarchenko [11, 15, 31], corresponds to our approach).

\footnotetext{
* corresponding author; e-mail: pak@math.su.se
}

Let us note that the inverse problem we are discussing is not just the problem to recover the potential in the Schrödinger equation but contains three entirely connected sub-problems:

- reconstruction of the underlying metric graph;

- calculation of the potential in the Schrödinger operator:

- determining the matching conditions at the internal vertices.

Our main focus will be on the difference between inverse problems for trees (graphs without cycles) and graphs with nontrivial topology (containing cycles and loops). It appears that for trees the inverse problem can be solved using just the TW-matrix. The main technical tool we use is so-called boundary control method [8]. In the case where cycles are present the knowledge of the TW-matrix is not enough to reconstruct the potential on the cycle, while potential on the branches can be calculated using the boundary control method as in the case of trees. The same method allows one to reconstruct the branches of the graph which can be seen as metric trees. It was suggested in [23] to extend the set of spectral data by considering the TW-matrices for magnetic Schrödinger operators. The corresponding TW-matrices do not depend on the concrete form of the magnetic potential, but on the fluxes of the magnetic field through the cycles. The corresponding inverse problem was first solved for graphs having just one cycle and standard matching conditions at the internal vertices [23]. This approach can easily be generalized for the case of compact graphs with several cycles [26].

Our attempts to investigate the third inverse problem to determine the matching conditions leads to interesting observations that the solvability of the potential inverse problem depends on the matching conditions in an unexpected way, namely that for certain classes of matching conditions the potential on the cycle is uniquely determined by just one TW-matrix (corresponding to zero 
magnetic potential) [27]. Reconstruction of the metric graph has not been discussed in detail yet. One may just mention that the branches may be reconstructed using the leaf-peeling method developed originally for trees. The Euler characteristic of the graph can be seen from the spectrum of the Schrödinger operator with zero magnetic potential [21, 22]. Graph's Albanese torus characterizing how different elementary cycles are connected to each other may also be seen from the spectra of the magnetic operators [35]. In the case the lengths of the graph are rationally independent the inverse problem can be solved using just one spectrum $[18,19]$. Other special classes of graphs have been studied in $[9,16,17,20,34]$.

The goal of this paper is to give an introduction into the area of inverse problems for quantum graphs describing first the leaf-peeling procedure. In the last section we explain in detail why this method cannot be generalized for graphs with cycles. We describe how does magnetic field help to solve the inverse problem in that case and what is the role of matching conditions. The second section is devoted to definitions.

\section{Titchmarsh-Weyl function for quantum graphs and inverse problems}

\subsection{Magnetic Schrödinger operator}

Let $\Gamma$ be a metric graph - collection of edges $\Delta_{n}=$ $\left[x_{2 n-1}, x_{2 n}\right], n=1,2, \ldots, N$ connected at the vertices $V_{m}, m=1,2, \ldots, M$, which can be considered as equivalence classes in the set $\boldsymbol{V}=\left\{x_{j}\right\}_{j=1}^{2 N}$ of all end points. The magnetic Schrödinger operator is defined by two real potentials, electric (square integrable) and magnetic (continuous) respectively,

$$
q \in L_{1}(\Gamma), \quad a \in C(\Gamma), \quad q(x), a(x) \in \mathbb{R},
$$

as follows:

$$
L_{q, a}=\left[\mathrm{i} \frac{\mathrm{d}}{\mathrm{d} x}+a(x)\right]^{2}+q(x) .
$$

The domain of the differential operator contains all functions from the Sobolev space $W_{2}^{2}\left(\Gamma \cup_{m=1}^{M} V_{m}\right)$, so that the range belongs to the Hilbert space $L_{2}(\Gamma)$.

In order to make the operator self-adjoint one has to restrict its domain further by introducing extra boundary/ matching conditions. Let us denote by $\partial \Gamma$ the boundary of $\Gamma$ - the set of all vertices of degree 1 . All other vertices (of degree greater or equal to 2) will be called internal. Let us denote by $\boldsymbol{u}^{m}, \partial \boldsymbol{u}^{m}$ the vectors of boundary values of the function $u$ and its extended normal derivative at the vertex $V_{m}$. These vectors have dimension equal to the degree of the vertex. The extended normal derivative is determined by the following equality:

$$
\partial u\left(x_{j}\right)=\left\{\begin{array}{c}
\lim _{x \rightarrow x_{j}}\left(\frac{\mathrm{d}}{\mathrm{d} x} u(x)-\mathrm{i} a(x) u(x)\right), \\
x_{j} \text { is the left end point, } \\
-\lim _{x \rightarrow x_{j}}\left(\frac{\mathrm{d}}{\mathrm{d} x} u(x)-\mathrm{i} a(x) u(x)\right), \\
x_{j} \text { is the right end point. }
\end{array}\right.
$$

Then the most general matching conditions at internal vertices can be written in the form

$$
\mathrm{i}\left(S_{m}-I\right) \boldsymbol{u}^{m}=\left(S_{m}+I\right) \partial \boldsymbol{u}^{m},
$$

where $S_{m}$ is a unitary matrix. We assume that it is irreducible, i.e. it cannot be transformed to a block-diagonal form by permutations. We assume that the functions from the domain of the operator satisfy the Dirichlet boundary conditions on graph's boundary

$$
\left.u\right|_{\partial \Gamma}=0 \text {. }
$$

So defined differential operator is self-adjoint and will be denoted by $L_{q, a}^{S}(\Gamma)$ in what follows.

Let us note that the role of matching conditions is not only to make the operator self-adjoint, but to connect the edges meeting at the corresponding vertex. With every internal vertex $V_{m}$ we associate the vertex scattering matrix

$$
S_{m}(\lambda)=\frac{(k+1) S_{m}+(k-1) I}{(k-1) S_{m}+(k+1)}, \quad \lambda=k^{2},
$$

so that $S_{m}=S_{m}(1)$. The high energy limit $S_{m}^{\infty}=$ $\lim _{\lambda \rightarrow \infty} S_{m}(\lambda)$ is going to play an important role in our consideration. This is a unitary Hermitian matrix and therefore its spectrum consists of \pm 1 . In the case the matrix $S_{m}$ is unitary Hermitian the vertex scattering matrix is independent of the energy $S_{m}(\lambda) \equiv S_{m}$ and coincides with the limit scattering matrix $S_{m}^{\infty}=S_{m}$.

So-called standard matching conditions (the function is continuous at the vertex and the sum of extended normal derivatives is zero) can also be described using our parameterization and correspond to

$$
\left(S_{m}\right)_{i j}= \begin{cases}-1+2 / \operatorname{deg} V_{m}, & i=j, \\ 2 / \operatorname{deg} V_{m}, & i \neq j .\end{cases}
$$

We exclude the case of standard boundary conditions for vertices of degree 2 (two intervals joined together) since in this case the two intervals can be substituted by one longer interval without any additional conditions at the point where the degree two vertex was situated.

\subsection{Titchmarsh-Weyl matrix function}

Our approach to inverse problem uses the TW-function as input data. To define the TW-function consider any solution to the differential equation

$$
L_{q, a} u=\lambda u, \quad \Im \lambda>0,
$$

satisfying matching conditions (2.4) at all internal vertices, but not necessarily on the boundary. The function $u=u(x, \lambda)$ is uniquely determined by its values on $\partial \Gamma$. The map

$$
M_{L_{q, a}^{S}(\Gamma)}(\lambda):\left.\left.u\right|_{\partial \Gamma} \mapsto \partial u\right|_{\partial \Gamma}
$$

is called the Titchmarsh-Weyl function for the quantum graph $L_{q, a}^{S}(\Gamma)$. It is a Nevanlinna type matrix function. Its singularities determine the spectrum of the operator $L_{q, a}^{S}$ (with the Dirichlet boundary conditions).

In the boundary control approach one uses the dynamical response operator $\boldsymbol{R}$, which can be defined as follows. Consider the following wave equation:

$$
\frac{\partial^{2}}{\partial t^{2}} w(x, t)+L_{q, a} w=0, \quad x \in \Gamma, \quad t>0,
$$




$$
\left\{\begin{array}{l}
w(x, 0)=\frac{\partial}{\partial t} w(x, 0) \equiv 0 \\
\left.w\right|_{\partial \Gamma}=F(t)
\end{array}\right.
$$

Here $F(t)$ is a vector valued function called boundary control. The dynamical response operator (the dynamical Dirichlet-to-Neumann map) is then defined by the equality:

$$
(\boldsymbol{R} F)=\left.\partial w\right|_{\partial \Gamma} .
$$

The TW-matrix and the dynamical response operator are related via Laplace transform as follows:

$$
\widehat{(\boldsymbol{R F})}(s)=\boldsymbol{M}\left(-s^{2}\right) \hat{F}(s) .
$$

The last equality implies that the sets of spectral data consisting of the TW-matrix and of the dynamical response operator are equivalent. Investigating the inverse problem for trees we are going to use these sets of spectral data simultaneously.

\section{Inverse problems for trees}

Inverse problems for the Schrödinger operators on compact metric trees have been considered by different authors $[1,2,5-7,10,12,37,38]$. The main object of studies was recovering of the potential on the edges. The question how to reconstruct the matching conditions at internal edges was discussed in [2, 12], but complete solution to all three inverse problems (including reconstruction of the underlying metric tree) has never been presented. Our plan in this section is to give a sketch how to solve this inverse problem, details will be published in [3].

Discussing the inverse problem for trees one should take into account the following observations:

Remark 1. Let $L_{q}^{S}$ be a Schrödinger operator on a metric graph $\Gamma$. Let $\theta(x)$ be a real-valued function on $\Gamma$, constant on each interval $\Delta_{n}$ and equal to zero on all boundary edges:

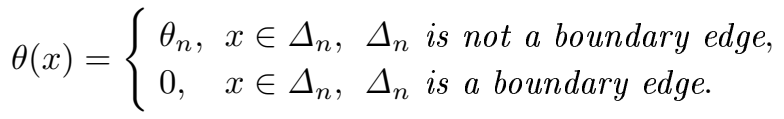

Then the similarity transformation

$$
L_{q} \rightarrow L_{q}(\theta)=\mathrm{e}^{-\mathrm{i} \theta(x)} L_{q} \mathrm{e}^{\mathrm{i} \theta(x)}
$$

preserves the Titchmarsh-Weyl function. In other words, the operators $L_{q}$ and $L_{q}(\theta)$ have precisely the same Titchmarsh-Weyl matrices. It follows that the inverse problem can be solved only up to the similarity transformation (3.1). Let us note that this transformation does not change the graph $\Gamma$ and the potential $q$ but affects the matching conditions only.

Remark 2. If the vertex scattering matrix parameterizing matching conditions has zero entries, then it might happen that the metric graph cannot be reconstructed uniquely. Such a counterexample was first presented in [25], where the Laplace operator $L^{S}$ on the cross graph depicted in Fig. 1 was considered. If the vertex scattering matrix $S_{1}$ associated with the central vertex $V_{1}=\left\{x_{2}, x_{4}, x_{6}, x_{8}\right\}$ is chosen such that there is no transition between the opposite branches of the cross and no reflection from the central vertex, then all crosses with equal distances between the "neighboring" boundary vertices $d\left(x_{1}, x_{3}\right), d\left(x_{3}, x_{5}\right), d\left(x_{5}, x_{7}\right), d\left(x_{7}, x_{1}\right)$ have identical TW-matrices.

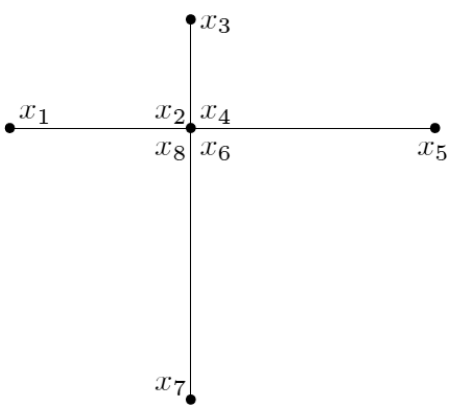

Fig. 1. Metric cross.

The matrix $S_{1}$ possessing described properties has the form

$$
\begin{aligned}
& S_{1}=\left(\begin{array}{cccc}
0 & \alpha & 0 & \beta \\
\alpha & 0 & \sigma \beta & 0 \\
0 & \sigma \beta & 0 & -\sigma \alpha \\
\beta & 0 & -\sigma \alpha & 0
\end{array}\right), \quad \alpha^{2}+\beta^{2}=1, \\
& \alpha, \beta \in \mathbb{R}, \quad \sigma= \pm 1,
\end{aligned}
$$

and the matching conditions can be written as

$$
\begin{aligned}
& \left(\begin{array}{cccc}
-1 & \alpha & 0 & \beta \\
\alpha & -1 & \sigma \beta & 0
\end{array}\right)\left(\begin{array}{l}
u\left(x_{2}\right) \\
u\left(x_{4}\right) \\
u\left(x_{6}\right) \\
u\left(x_{8}\right)
\end{array}\right)=\mathbf{0}, \\
& \left(\begin{array}{cccc}
1 & \alpha & 0 & \beta \\
\alpha & 1 & \sigma \beta & 0
\end{array}\right)\left(\begin{array}{l}
u^{\prime}\left(x_{2}\right) \\
u^{\prime}\left(x_{4}\right) \\
u^{\prime}\left(x_{6}\right) \\
u^{\prime}\left(x_{8}\right)
\end{array}\right)=\mathbf{0} .
\end{aligned}
$$

It follows that to ensure that the metric graph is uniquely determined by the TW-matrix one has to require that the vertex scattering matrices $S_{m}(k)$ do not have identically vanishing entries.

Remark 3. As we already mentioned, degree two vertices should be ignored in the case of standard matching conditions, since the corresponding two edges can be substituted by one bigger edge without changing the spectral data for the whole graph. These matching conditions will be excluded by requiring that $S_{m}(\lambda)$ do not have identically equal to zero entries.

Remark 4. In order to simplify the reconstruction of matching conditions we are going to assume that not only the matrices $S_{m}$, but also the matrices $S_{m}^{\infty}$ are irreducible. Irreducibility of $S_{m}$ is a necessary condition, since reducible matrices correspond to graphs with some connections between the edges broken.

The following theorem can be proven [3]:

Theorem 5. Let $\Gamma$ be a compact metric tree formed by a finite number of edges. Let $L_{q}^{S}=-\frac{\mathrm{d}^{2}}{\mathrm{~d} x^{2}}+q(x)$ be a 
Schrödinger operator in $L_{2}(\Gamma)$ defined on the functions satisfying the matching conditions (3.2) at all internal vertices and the Dirichlet boundary conditions on $\partial \Gamma$. Assume that the potential $q$ is real valued and belongs to $L_{1}(\Gamma)$. Assume that the vertex scattering matrices $S_{m}(\lambda), m=1,2, \ldots, M$ do not have identically equal to zero entries and the matrices $S_{m}$ as well $S_{m}^{\infty}$ are irreducible. Then the TW-matrix associated with the graph boundary determines the quantum graph, i.e. the unique metric graph $\Gamma$, the unique real potential $q$ and the matching conditions up to the transformation described by (3.1).

In the proof we are going to use the fact that there exists explicit formula (2.10) connecting the TW-matrix $M$ and the dynamical response operator $\boldsymbol{R}$ for the wave equation evolution on the graph. This would allow us to use the boundary control method $[4,8,36]$. The quantum tree will be recovered by leaf-peeling method proposed first in [1], where the tree is recovered locally by taking away boundary edges and thus reducing the inverse problem to a smaller and smaller graph.

\subsection{Recovering metric tree}

Let us pick up any boundary vertex. Our first goal is to reconstruct the neighboring edge and the whole sheaf of boundary edges attached to the same internal vertex. Every edge is uniquely determined by its length, so we need to determine the boundary vertices corresponding to the sheaf and the lengths of the edges in the sheaf.

The lengths of all boundary edges can be determined from the diagonal part of the response operator. Let $\left\{x_{2 j-1}\right\}$ be a boundary vertex and let us denote the corresponding boundary edge by $\Delta_{j}=\left[x_{2 j}, x_{2 j-1}\right]$. The root vertex for the sheaf will be denoted by $V_{m}, m=$ $m\left(x_{2 j-1}\right)$. One can show that the diagonal part of the response operator possesses the following representation:

$$
\begin{aligned}
& \left(\boldsymbol{R} f \boldsymbol{e}_{2 j-1}\right)_{2 j-1}(t) \\
& =-\frac{\mathrm{d}}{\mathrm{d} t} f(t)+2 P_{\Delta_{j}} S_{m}^{\infty} P_{\Delta_{2 j-1}} \frac{\mathrm{d}}{\mathrm{d} t} f\left(t-2 \ell_{j}\right) \\
& -4 P_{\Delta_{j}} H_{m} P_{N_{-1}^{m}}^{\perp} P_{\Delta_{j}} f\left(t-2 \ell_{j}\right)+\text { convolution } \\
& \text { operator with } L_{1, \text { loc }} \text { kernel, } \quad 0 \leq t<3 \ell_{j} .
\end{aligned}
$$

In the last formula we used the following notations:

- $\boldsymbol{e}_{2 j-1}$ the standard basis vector in $\mathbb{C}^{\operatorname{dim}} \partial \Gamma$,

- $S_{m}$ - the vertex scattering matrix parameterizing the matching conditions at the vertex $V_{m}$,

- $S_{m}^{\infty}=\lim _{k \rightarrow \infty} \frac{(k+1) S_{m}+(k-1) I}{(k-1) S_{m}+(k+1) I}=-P_{N_{-1}^{m}}+P_{N_{-1}^{m}}^{\perp}$,

- $H_{m}=\mathrm{i} \frac{S_{n}-I}{S_{m}+I} P_{N_{-1}}^{\perp}$,

- $P_{\Delta_{j}}$ is the orthogonal project in $\mathbb{C}^{\operatorname{deg} V_{m}}$ on the basis vector corresponding to the edge $\Delta_{j}$.

Writing the whole response operator as a convolution with a generalized kernel, we see that the kernel has singularities with the support at $t=2 \ell_{j}$ :

$$
2 P_{\Delta_{j}} S_{m}^{\infty} P_{\Delta_{j}} \delta^{\prime}\left(t-2 \ell_{j}\right)-4 P_{\Delta_{j}} H_{m} P_{N_{-1}}^{\perp} P_{\Delta_{j}} \delta\left(t-2 \ell_{j}\right) .
$$

Since the vertex scattering matrix $S_{m}(k)$ does not have identically equal to zero entries, at least one of the entries $\left(S_{m}^{\infty}\right)_{j j}$ or $\left(H_{m} P_{N_{-1}}^{\perp}\right)_{j j}$ is different from zero. It follows that the length $\ell_{j}$ of the boundary edge can be calculated from the corresponding diagonal entry of the response operator.

Assume now that the lengths of all boundary edges are determined. Since all entries of the vertex scattering matrices are not identically equal to zero, the traveling times between different boundary vertices are equal precisely to the distances between these vertices on the graph $\Gamma$. The wave equation along the edges have unit propagation speed and nonzero entries of $S_{m}(k)$ guarantee that waves penetrate through all vertices without any time delay. It follows that we can now identify all edges forming sheafs: two edges $\Delta_{j}$ and $\Delta_{i}$ belong to the same sheaf if and only if $\ell_{j}+\ell_{i}$ is equal to the distance between the boundary points $x_{2 j-1}$ and $x_{2 i-1}$. If the distance is equal to $\ell_{j}$ (and also to $\ell_{i}$ ) then the graph consists of just one edge connecting directly the two boundary points. If $\ell_{j}+\ell_{i}$ for all $i$ is less than the corresponding distance, then the edge $\Delta_{j}$ is connected to an internal vertex of degree 2, i.e. the sheaf consists of just one edge.

We may assume now that a certain sheaf in $\Gamma$ is reconstructed, which means that we know which boundary points belong to the sheaf and the lengths of the corresponding edges.*

\subsection{Recovering potential}

Recovering of the potential can be done using standard boundary control method [8, 36] as described in [1]. For small values of $t, t<\ell$, the diagonal part of the response operator $^{\dagger}$ depends on the potential on the corresponding boundary edge only $[1,36]$

$$
\begin{aligned}
& \left(\boldsymbol{R} f \boldsymbol{e}_{2 j-1}\right)_{2 j-1}(t)=-f^{\prime}(t)+\int_{0}^{t} r(t-\tau) f(\tau) \mathrm{d} \tau, \\
& \quad 0 \leq t<2 \ell_{j},
\end{aligned}
$$

with $r(\cdot)=\partial_{x} w(0, \cdot)$, where $w(x, t)$ is a solution to the Goursat problem

$$
\left\{\begin{array}{l}
{\left[-\frac{\partial^{2}}{\partial x^{2}}+q(x)\right] w=-\frac{\partial^{2}}{\partial t^{2}} w} \\
w\left(x_{2 j-1}, t\right)=0 \\
w\left(x, x-x_{2 j-1}\right)=-\frac{1}{2} \int_{x_{2 j-1}}^{x} q(y) \mathrm{d} y .
\end{array}\right.
$$

\footnotetext{
* In the case where the graph $\Gamma$ has no vertices of degree 2 the whole tree may be reconstructed at once using distances between the boundary edges, see Lemma 2.12 from [35].

$\dagger$ Of course we consider here the diagonal part corresponding to end points associated with the sheaf.
} 
It follows that the diagonal entry of the response operator determines the kernel $r(t), t \in\left[0,2 \ell_{j}\right]$ and therefore allows one to calculate $w(x, t)$ and therefore to reconstruct the potential $q$ on each boundary edge from the sheaf.

From now on we may assume that not only the sheaf, but also the potential on it is known. To perform the leaf-peeling it remains to determine the matching conditions at the vertex $V_{m}$.

\subsection{Recovering matching conditions}

As proposed in [2] matching conditions at the root vertex for the sheaf can be determined using the following trick: let us substitute the original graph $\Gamma$ with the graph $\tilde{\Gamma}$ obtained from $\Gamma$ by substituting the sheaf in question with the sheaf formed by precisely the same number of edges having equal length $\ell$. The potential $q$ is extended by zero to the new sheaf but is preserved on the remaining part of the graph. Then the operator $L_{q}^{S}(\tilde{\Gamma})$ is determined by the same matching conditions at all internal vertices. It appears that the TW-matrices for the operators $L_{q}^{S}(\Gamma)$ and $L_{q}^{S}(\tilde{\Gamma})$ are in one-to-one correspondence.

Assume without loss of generality that the boundary edges $\Delta_{1}, \Delta_{2}, \ldots, \Delta_{n}$ form the sheaf. Then the root vertex $V_{1}$ is a union of the end points $V_{1}=$ $\left\{x_{2}, x_{4}, \ldots, x_{2 n+2}\right\}$, where we denoted by $x_{2 n+2}$ the end point which does not belong to the sheaf, but is contained in $V_{1}$. The lengths of the edges in the sheaf and potential there are already known. Consider any vector $\left.u\right|_{\partial \Gamma}$ of boundary values, then the normal derivatives for the solution of (2.6) are determined by the TW-function

$$
\left.\partial_{n} u\right|_{\partial \Gamma}=\left.M_{L_{q}^{S}(\Gamma)}(\lambda) u\right|_{\partial \Gamma} .
$$

Then the function $u$ on all boundary edges from the sheaf is a solution to the equation

$$
-u^{\prime \prime}+q(x) u=\lambda u
$$

and therefore is uniquely determined by the Cauchy data $u\left(x_{2 j-1}\right), u^{\prime}\left(x_{2 j-1}\right)$. This allows us to determine the boundary values at sheaf's internal vertex $u\left(x_{2 j}\right)$, $u^{\prime}\left(x_{2 j}\right), j=1,2, \ldots, n$. Consider now the operator $L_{q}^{S}(\tilde{\Gamma})$ and the corresponding solution $\tilde{u}=\tilde{u}(x, \lambda)$. We choose $\tilde{u}(x)=u(x)$, whenever $x$ does not belong to the sheaf in question. The function $\tilde{u}$ is extended to the whole $\tilde{\Gamma}$ by solving the Helmholtz equation $-\tilde{u}^{\prime \prime}(x)=\lambda \tilde{u}$ on the sheaf with the same boundary values at $x_{2 j}, j=$ $1,2, \ldots, n$ as the function $u$ :

$$
\begin{aligned}
& \tilde{u}\left(x_{2 j}\right)=u\left(x_{2 j}\right), \\
& \quad \tilde{u}^{\prime}\left(x_{2 j}\right)=u^{\prime}\left(x_{2 j}\right), \quad j=1,2, \ldots, n .
\end{aligned}
$$

The function $\tilde{u}$ is defined now on the whole $\tilde{\Gamma}$ and satisfies matching conditions at all internal vertices.

Consider the vector of boundary values $\left.\tilde{u}\right|_{\partial \tilde{\Gamma}},\left.\partial_{n} \tilde{u}\right|_{\partial \tilde{\Gamma}}$ connected via the TW-matrix for $\tilde{\Gamma}$ :

$$
\left.\partial_{n} \tilde{u}\right|_{\partial \tilde{\Gamma}}=\left.M_{L_{q}^{S}(\tilde{\Gamma})}(\lambda) \tilde{u}\right|_{\partial \tilde{\Gamma}} \cdot
$$

The map $\left.\left.u\right|_{\partial \Gamma} \rightarrow \tilde{u}\right|_{\partial \tilde{\Gamma}}$ is a map between the vector spaces of the same dimension and obviously onto. It follows that Eq. (3.6) determines $M_{L_{q}^{S}(\tilde{\Gamma})}(\lambda)$.

In Ref. [2] it was shown that the principal $n \times n$ block of the response operator for the star graph determines the matching conditions at the central vertex up to the transformation described in Remark 1. This result can be generalized to our case by taking

$$
\theta(x)= \begin{cases}1, & \text { on all boundary edges }, \\ \theta_{\mathrm{int}}, & \text { on all internal edges. }\end{cases}
$$

Since the procedure is local, possibly complicated form of the graph does not affect it. In Ref. [2] it is assumed in addition that $S_{1}^{\infty}$ is irreducible - this assumption can be removed. The unitary matrix $S_{1}$ is then reconstructed up to the following transformation:

$$
S_{1} \rightarrow \operatorname{diag}\left(1,1, \ldots, 1, \theta_{\text {int }}\right)^{-1} S_{1} \operatorname{diag}\left(1,1, \ldots, 1, \theta_{\text {int }}\right) .
$$

We have now determined the sheaf, the real potential $q$ on it and the matching conditions at the neighboring internal vertex - the root vertex for the sheaf.

\subsection{Leaf-peeling procedure}

We are ready now to calculate the TW-matrix for the graph $\Gamma^{\prime}$ obtained from $\Gamma$ by peeling away the sheaf we reconstructed. Assume that the free parameter $\theta_{\text {int }}$ parameterizing the matching conditions is fixed

$$
\mathrm{i}\left(S_{1}-I\right)\left(\begin{array}{c}
u\left(x_{2}\right) \\
u\left(x_{4}\right) \\
\vdots \\
u\left(x_{2 n}\right) \\
u\left(x_{2 n+2}\right)
\end{array}\right)=-\left(S_{1}+I\right)\left(\begin{array}{c}
u^{\prime}\left(x_{2}\right) \\
u^{\prime}\left(x_{4}\right) \\
\vdots \\
u^{\prime}\left(x_{2 n}\right) \\
u^{\prime}\left(x_{2 n+2}\right)
\end{array}\right) .
$$

These matching conditions determine a certain $n+1$ dimensional (Lagrangian) plane in the $2(n+1)$-dimensional space of boundary values. This plane is not orthogonal to the plane spanned up by $u\left(x_{2 n+2}\right), u^{\prime}\left(x_{2 n+2}\right)$, since otherwise the matching conditions would be reducible. It follows that the values $u\left(x_{2 n+2}\right), u^{\prime}\left(x_{2 n+2}\right)$ are uniquely determined by $u\left(x_{2 j}\right), u^{\prime}\left(x_{2 j}\right), j=1,2, \ldots, n$ and one may choose $u\left(x_{2 j}\right), j=1,2, \ldots, n$ so that $u\left(x_{2 n+2}\right) \neq 0$.

We conclude that the TW-function for the graph $\Gamma$ determines the TW-function for the graph $\Gamma^{\prime}$. The inverse problem is reduced to the inverse problem for a smaller graph. Since the graph $\Gamma$ is finite, this procedure may be continued, but it ends after a finite number of steps.

It is clear that our assumptions on the parameters are far from being optimal. Most probably the requirement that the matrices $S_{m}^{\infty}$ are irreducible may be waived. It is not necessary to require that all entries of all vertex scattering matrices $S_{m}(k)$ are not identically equal to zero. For example in the case of cross one may allow that the transition amplitudes to the opposite edges are equal to zero. Then the lengths of the edges are determined looking at the waves reflected from the central vertex. For more complicated trees even more freedom may be observed. 


\section{Inverse problems for graphs with cycles}

Our aim in this section is to describe difficulties which appear in solving the inverse problem for graphs with cycles. The case of just one cycle was considered in [23, 24]. The branches of the graph can be reconstructed using the boundary control method and the leaf-peeling procedure. Here it is very important that this procedure is local and allows one to recover just a certain part of the metric graph and potential on it from the corresponding part of the TW-matrix.

It remains to recover the potential on the cycle and this problem appears much more difficult than the inverse problem for trees. One TW-function is not enough to recover the potential there. One needs to increase the set of spectral data. In Ref. [23] it was suggested to consider magnetic Schrödinger operators and as the set of spectral data to take the TW-function depending on the flux of the magnetic field through the cycle. This problem was analyzed in the case of standard matching conditions. For a loop (cycle formed by just one edge) the potential on it in general is not reconstructed uniquely. If the cycle is formed by several edges, then the potential on it is reconstructable, except for a few very special counterexamples $[23,24]$. On the other hand, the loop with two attached wires was studied in [27]. It was shown that for very special (not standard) matching conditions the potential on the loop is reconstructable.

The aim of this section is to study whether for general matching conditions the potential on the loop is reconstructable from the corresponding TW-function considered for different values of the magnetic flux. We give a partial answer to this question considering a loop with one wire attached and real Hermitian matching conditions at the vertex.

Consider the lasso graph depicted in Fig. 2.

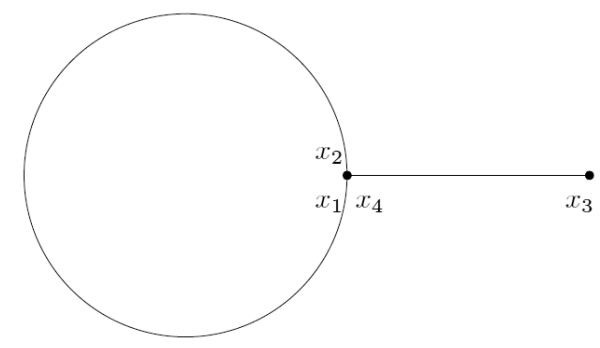

Fig. 2. Lasso graph $\Gamma_{2}$.

The transfer matrix $T$ for the Schödinger equation $-u^{\prime \prime}+q(x) u=k^{2} u$ on the interval $\left[x_{1}, x_{2}\right]$ will be used

$$
T(k):\left(\begin{array}{c}
u\left(x_{1}\right) \\
u^{\prime}\left(x_{1}\right)
\end{array}\right) \mapsto\left(\begin{array}{c}
u\left(x_{2}\right) \\
u^{\prime}\left(x_{2}\right)
\end{array}\right) .
$$

All entries of the transfer matrix are analytic functions of exponential type having special asymptotics [32], the transfer matrix has unit determinant. In order to reconstruct potential on the loop one needs to know the functions $t_{12}$ and $t_{22}$. The zeroes of these functions determine the Dirichlet-Dirichlet and Dirichlet-Neumann spectra for the Schrödinger operator on the interval $\left[x_{1}, x_{2}\right]$ [28]. On the other hand, these functions as functions of exponential type with prescribed asymptotics are uniquely determined by their zeros.

If the matching conditions at the central vertex are standard, then the TW-function associated with the ring is given by

$$
\begin{aligned}
& M_{\Phi}\left(\lambda, \Gamma_{1}\right):=\frac{\psi^{\prime}\left(x_{1}\right)-\psi^{\prime}\left(x_{2}\right)}{\psi\left(x_{1}\right)} \\
& =\frac{\hat{\psi}^{\prime}\left(x_{1}\right)-\mathrm{e}^{\mathrm{i} \phi_{1}} \hat{\psi}^{\prime}\left(x_{2}\right)}{\hat{\psi}\left(x_{1}\right)}=\frac{2 \cos \Phi-\operatorname{Tr} T(k)}{t_{12}(k)} .
\end{aligned}
$$

It follows that just the trace of the transfer matrix $\operatorname{Tr} T=$ $t_{11}+t_{22}$ and the coefficient $t_{12}$ are determined by $M$. The potential on the loop is not uniquely determined. The corresponding formula contains in general infinitely many parameters [23].

Let us study the case of arbitrary properly connecting real matching conditions leading to energy independent vertex scattering matrices. In other words, we assume that the functions from the domain of the operator satisfy the following matching conditions:

$$
\mathrm{i}(S-I)\left(\begin{array}{c}
u\left(x_{1}\right) \\
u\left(x_{2}\right) \\
u\left(x_{4}\right)
\end{array}\right)=(S+I)\left(\begin{array}{c}
u^{\prime}\left(x_{1}\right) \\
-u^{\prime}\left(x_{2}\right) \\
-u\left(x_{4}\right)^{\prime}
\end{array}\right),
$$

where $S$ is a real Hermitian unitary irreducible matrix. Every such matrix can be written in the form

$$
S=\left(\begin{array}{ccc}
\alpha & \beta & \gamma \\
\beta & \delta & \epsilon \\
\gamma & \epsilon & \zeta
\end{array}\right), \quad \alpha, \beta, \gamma, \delta, \epsilon, \zeta \in \mathbb{R} .
$$

The parameters satisfy obvious conditions to ensure that the matrix is unitary.

Observe that the nondiagonal entries of $S$ cannot be equal to zero, since otherwise the matrix will be reducible. Assume without loss of generality that $\gamma=0$. It follows then that either $\beta$ or $\epsilon$ should also be zero to ensure that the first and last rows are orthogonal. The corresponding matrices are reducible, but reducible matrices are excluded from our consideration.

Our immediate goal is to calculate the TW-function for the loop graph and described matching conditions. The values of the function on the loop are related via the corresponding transfer matrix (4.1). The boundary values $u\left(x_{4}\right)$ and $u^{\prime}\left(x_{4}\right)$ are connected via the TW-function, which is to be calculated

$$
u^{\prime}\left(x_{4}\right)=M(\lambda) u\left(x_{4}\right) .
$$

Substituting equalities (4.4) and (4.1) into the matching conditions (4.3) we get the following linear equation: 


$$
\left[\mathrm{i}(S-I)\left(\begin{array}{ccc}
1 & 0 & 0 \\
t_{11} & t_{12} & 0 \\
0 & 0 & 1
\end{array}\right)+(S+I)\left(\begin{array}{ccc}
0 & -1 & 0 \\
t_{21} & t_{22} & 0 \\
0 & 0 & M
\end{array}\right)\right]\left(\begin{array}{c}
u\left(x_{1}\right) \\
u^{\prime}\left(x_{1}\right) \\
u\left(x_{4}\right)
\end{array}\right)=0,
$$

which has a nontrivial solution if and only if the determinant of the matrix is equal to zero

$$
\operatorname{det}\left[\mathrm{i}(S-I)\left(\begin{array}{ccc}
1 & 0 & 0 \\
t_{11} & t_{12} & 0 \\
0 & 0 & 1
\end{array}\right)+(S+I)\left(\begin{array}{ccc}
0 & -1 & 0 \\
t_{21} & t_{22} & 0 \\
0 & 0 & M
\end{array}\right)\right]=0
$$

Similar formula holds in the case of nonzero magnetic potential, which can be removed on the interval $\left[x_{1}, x_{2}\right]$, but results in multiplication of the transfer matrix coefficients by $\mathrm{e}^{\mathrm{i} \Phi}$, where $\Phi$ is the integral of the magnetic potential over the interval $\Phi=\int_{x_{1}}^{x_{2}} a(x) \mathrm{d} x$. The corresponding formula reads as follows:

$$
\operatorname{det}\left[\mathrm{i}(S-I)\left(\begin{array}{ccc}
1 & 0 & 0 \\
\mathrm{e}^{\mathrm{i} \Phi} t_{11} & \mathrm{e}^{\mathrm{i} \Phi} t_{12} & 0 \\
0 & 0 & 1
\end{array}\right)+(S+I)\left(\begin{array}{ccc}
0 & -1 & 0 \\
\mathrm{e}^{\mathrm{i} \Phi} t_{21} & \mathrm{e}^{\mathrm{i} \Phi} t_{22} & 0 \\
0 & 0 & M(\Phi)
\end{array}\right)\right]=0,
$$

or in a more detailed form

$$
\operatorname{det}\left[\mathrm{i}\left(\begin{array}{ccc}
\alpha-1+\beta t_{11} & \beta t_{12} & \gamma \\
\beta+(\delta-1) t_{11} & (\delta-1) t_{12} & \epsilon \\
\gamma+\epsilon t_{11} & \epsilon t_{12} & \zeta-1
\end{array}\right)+\left(\begin{array}{ccc}
\beta t_{21} & -(\alpha+1)+\beta t_{22} & \gamma M \\
(\delta+1) t_{21} & -\beta+(\delta+1) t_{22} & \epsilon M \\
\epsilon t_{21} & -\gamma+\epsilon t_{22} & (\zeta+1) M
\end{array}\right)\right]=0 .
$$

This equation determines the TW-function $M=M(\lambda, \Phi)$, but the corresponding formula is rather involved. We are interested in its dependence upon the phase $\Phi$ which suggests us to write this formula in the form

$$
M(\lambda)=-\frac{a_{0}+a_{1} \mathrm{e}^{\mathrm{i} \Phi}+a_{2} \mathrm{e}^{2 \mathrm{i} \Phi}}{b_{0}+b_{1} \mathrm{e}^{\mathrm{i} \Phi}+b_{2} \mathrm{e}^{2 \mathrm{i} \Phi}},
$$

where

$$
\begin{aligned}
& a_{0}=\operatorname{det}\left(\begin{array}{ccc}
\alpha-1 & \alpha+1 & \gamma \\
\beta & \beta & \epsilon \\
\gamma & \gamma & \zeta-1
\end{array}\right)=2[\epsilon \gamma-\beta(\zeta-1)] ; \\
& a_{1}=t_{11} \operatorname{det}\left(\begin{array}{ccc}
\beta & \alpha+1 & \gamma \\
\delta-1 & \beta & \epsilon \\
\epsilon & \gamma & \zeta-1
\end{array}\right)-\mathrm{i} t_{21} \operatorname{det}\left(\begin{array}{ccc}
\beta & \alpha+1 & \gamma \\
\delta+1 & \beta & \epsilon \\
\epsilon & \gamma & \zeta-1
\end{array}\right)-\mathrm{i} t_{12} \operatorname{det}\left(\begin{array}{ccc}
\alpha-1 & \beta+1 & \gamma \\
\beta & \delta-1 & \epsilon \\
\gamma & \epsilon & \zeta-1
\end{array}\right) \\
& -t_{22} \operatorname{det}\left(\begin{array}{ccc}
\alpha-1 & \beta+1 & \gamma \\
\beta & \delta+1 & \epsilon \\
\gamma & \epsilon & \zeta-1
\end{array}\right)=t_{11}\left[\beta^{2}(\zeta-1)+\gamma^{2}(\delta-1)+\epsilon^{2}(\alpha+1)-2 \beta \gamma \epsilon-(\alpha+1)(\delta-1)(\zeta-1)\right] \\
& +t_{22}\left[\beta^{2}(\zeta-1)+\gamma^{2}(\delta+1)+\epsilon^{2}(\alpha-1)-2 \beta \gamma \epsilon-(\alpha-1)(\delta+1)(\zeta-1)\right]-\mathrm{i} t_{21}\left[\beta^{2}(\zeta-1)+\gamma^{2}(\delta+1)+\epsilon^{2}(\alpha+1)\right. \\
& -2 \beta \gamma \epsilon-(\alpha+1)(\delta+1)(\zeta-1)]+\mathrm{i} t_{12}\left[\beta^{2}(\zeta-1)+\gamma^{2}(\delta-1)+\epsilon^{2}(\alpha-1)-2 \beta \gamma \epsilon-(\alpha-1)(\delta-1)(\zeta-1)\right] \text {; } \\
& a_{2}=\operatorname{det}\left(\begin{array}{ccc}
\mathrm{i} \beta t_{11}+\beta t_{21} & \mathrm{i} \beta t_{12}+\beta t_{22} & \mathrm{i} \gamma \\
\mathrm{i}(\delta-1) t_{11}+(\delta+1) t_{21} & \mathrm{i}(\delta-1) t_{12}+(\delta+1) t_{22} & \mathrm{i} \epsilon \\
\mathrm{i} \epsilon t_{11}+\epsilon t_{21} & \mathrm{i} \epsilon t_{12}+\epsilon t_{22} & \mathrm{i}(\zeta-1)
\end{array}\right) \\
& =-\left(t_{11} t_{22}-t_{12} t_{21}\right) \operatorname{det}\left(\begin{array}{ccc}
\beta & \beta & \gamma \\
\delta-1 & \delta+1 & \epsilon \\
\epsilon & \epsilon & \zeta-1
\end{array}\right)=2[\epsilon \gamma-\beta(\zeta-1)] \\
& b_{0}=-\mathrm{i} \operatorname{det}\left(\begin{array}{ccc}
\alpha-1 & \alpha+1 & \gamma \\
\beta & \beta & \epsilon \\
\gamma & \gamma & \zeta+1
\end{array}\right)=-2 \mathrm{i}[\epsilon \gamma-\beta(\zeta+1)] \text {; }
\end{aligned}
$$




$$
\begin{aligned}
b_{1} & =-\mathrm{i} t_{11} \operatorname{det}\left(\begin{array}{ccc}
\beta & \alpha+1 & \gamma \\
\delta-1 & \beta & \epsilon \\
\epsilon & \gamma & \zeta+1
\end{array}\right)+t_{21} \operatorname{det}\left(\begin{array}{ccc}
\beta & \alpha+1 & \gamma \\
\delta+1 & \beta & \epsilon \\
\epsilon & \gamma & \zeta+1
\end{array}\right)-t_{12} \operatorname{det}\left(\begin{array}{ccc}
\alpha-1 & \beta & \gamma \\
\beta & \delta-1 & \epsilon \\
\gamma & \epsilon & \zeta+1
\end{array}\right) \\
& +\mathrm{i} t_{22} \operatorname{det}\left(\begin{array}{ccc}
\alpha-1 & \beta & \gamma \\
\beta & \delta+1 & \epsilon \\
\gamma & \epsilon & \zeta+1
\end{array}\right)=\mathrm{i} t_{11}\left[2 \beta \gamma \epsilon+(\alpha+1)(\delta-1)(\zeta+1)-\beta^{2}(\zeta+1)-\gamma^{2}(\delta-1)-\epsilon^{2}(\alpha+1)\right] \\
& +\mathrm{i} t_{22}\left[2 \beta \gamma \epsilon+(\alpha-1)(\delta+1)(\zeta+1)-\beta^{2}(\zeta+1)-\gamma^{2}(\delta+1)-\epsilon^{2}(\alpha-1)\right]+t_{21}[2 \beta \gamma \epsilon+(\alpha+1)(\delta+1)(\zeta+1) \\
& \left.-\beta^{2}(\zeta+1)-\gamma^{2}(\delta+1)-\epsilon^{2}(\alpha+1)\right]-t_{12}\left[2 \beta \gamma \epsilon+(\alpha-1)(\delta-1)(\zeta+1)-\beta^{2}(\zeta+1)-\gamma^{2}(\delta-1)-\epsilon^{2}(\alpha-1)\right] ; \\
b_{2} & =\operatorname{det}\left(\begin{array}{ccc}
\mathrm{i} \beta t_{11}+\beta t_{21} & \mathrm{i} \beta t_{12}+\beta t_{22} & \gamma \\
\mathrm{i}(\delta-1) t_{11}+(\delta+1) t_{21} & \mathrm{i}(\delta-1) t_{12}+(\delta+1) t_{22} & \epsilon \\
\mathrm{i} \epsilon t_{11}+\epsilon t_{21} & \mathrm{i} \epsilon t_{12}+\epsilon t_{22} & \zeta+1
\end{array}\right) \\
& \left.\quad \begin{array}{ccc}
\beta & \gamma & \gamma \\
\delta-1 & \delta+1 & \epsilon \\
\epsilon & \epsilon & \zeta+1
\end{array}\right)=-2 \mathrm{i}[\epsilon \gamma-\beta(\zeta+1)] .
\end{aligned}
$$

We see that $a_{0}=a_{2}$ and $b_{0}=b_{2}$ and formula (4.9) can be written in the form

$$
M(\lambda, \Phi)=-\frac{2 a_{0} \cos \Phi+a_{1}(\lambda)}{2 b_{0} \cos \Phi+b_{1}(\lambda)} .
$$

The TW-functions depend just on $\cos \Phi$, since the matching conditions are given by real Hermitian unitary matrix. The coefficients $a_{0}$ and $b_{0}$ are determined by $S$, then the functions $a_{1}(\lambda)$ and $b_{1}(\lambda)$ are uniquely determined by $M(\lambda, \Phi)$.

Considering $M(\lambda, 0)$ and $M(\lambda, \pi)$ we arrive at the following system of equations:

$$
\left\{\begin{array}{l}
a_{1}+M(\lambda, 0) b_{1}=-2\left[a_{0}+M(\lambda, 0) b_{0}\right], \\
a_{1}+M(\lambda, \pi) b_{1}=2\left[a_{0}+M(\lambda, \pi) b_{0}\right],
\end{array}\right.
$$

with the determinant $M(\lambda, \pi)-M(\lambda, 0)$. The TW-function $M(\lambda, \Phi)$ is independent of $\Phi$ if and only if

$$
a_{0} b_{1}(\lambda)=b_{0} a_{1}(\lambda), \quad \text { for all } \lambda \text {. }
$$

Remember that $a_{1}(\lambda)$ and $b_{1}(\lambda)$ are linear combinations of $t_{i j}(\lambda)$. The terms containing $t_{11}$ and $t_{22}$ cancel in (4.12). The asymptotics as $\lambda \rightarrow \infty$ is dominating by the function $t_{21}(\lambda) \sim-k \sin k\left(x_{2}-x_{1}\right)$. Comparing the corresponding coefficients we arrive at the following equality:

$$
\begin{aligned}
& {[\epsilon \gamma-\beta(\zeta-1)][2 \beta \gamma \epsilon+(\alpha+1)(\delta+1)(\zeta+1)} \\
& \left.\quad-\beta^{2}(\zeta+1)-\gamma^{2}(\delta+1)-\epsilon^{2}(\alpha+1)\right] \\
& \quad=[\epsilon \gamma-\beta(\zeta+1)][2 \beta \gamma \epsilon+(\alpha+1)(\delta+1)(\zeta-1) \\
& \left.\quad-\beta^{2}(\zeta-1)-\gamma^{2}(\delta+1)-\epsilon^{2}(\alpha+1)\right],
\end{aligned}
$$

which implies

$$
\begin{aligned}
& \epsilon \gamma(\alpha+1)(\delta+1) \\
& \quad+\beta^{2} \gamma \epsilon-\beta \gamma^{2}(\delta+1)-\beta \epsilon^{2}(\alpha+1)=0 .
\end{aligned}
$$

Eliminating $\epsilon \gamma, \gamma^{2}$ and $\epsilon^{2}$ using orthogonality and normalization of the row vectors in $S$ we obtain that

$$
\beta\left[(\alpha+1)(\delta+1)-\beta^{2}\right]=0 .
$$

Since $\beta$ cannot be equal to zero unless $S$ is reducible, we conclude that

$$
(\alpha+1)(\delta+1)-\beta^{2}=0
$$

must hold.

Under this condition even the terms containing $t_{21}$ cancel in (4.12), so only terms containing $t_{12}$ remain. Since $t_{12}(\lambda)$ is not identically equal to zero, (4.12) holds if and only if

$$
\begin{aligned}
& {[\epsilon \gamma-\beta(\zeta-1)][2 \beta \gamma \epsilon+(\alpha-1)(\delta-1)(\zeta+1)} \\
& \left.\quad-\beta^{2}(\zeta+1)-\gamma^{2}(\delta-1)-\epsilon^{2}(\alpha-1)\right] \\
& \quad=[\epsilon \gamma-\beta(\zeta+1)][2 \beta \gamma \epsilon+(\alpha-1)(\delta-1)(\zeta-1) \\
& \left.\quad-\beta^{2}(\zeta-1)-\gamma^{2}(\delta-1)-\epsilon^{2}(\alpha-1)\right] .
\end{aligned}
$$

Analysis similar to one already carried out for coefficients in front of $t_{21}$ leads to the second condition

$$
(\alpha-1)(\beta-1)-\beta^{2}=0 .
$$

Conditions (4.13) and (4.14) imply that the matrix $\left(\begin{array}{ll}\alpha & \beta \\ \beta & \delta\end{array}\right)$ has eigenvalues -1 and 1 , respectively. It follows that the unitary matrix $S$ containing this $2 \times 2$ matrix as the principle block is reducible, since the third eigenvector is just $(0,0,1)$. But reducible matrices are excluded from our consideration.

We conclude that the functions $M(\lambda, \Phi)$ and $M(\lambda, 0)$ determine $a_{1}(\lambda)$ and $b_{1}(\lambda)$.

It remains to study the question under which conditions the functions $t_{12}$ and $t_{22}$ can be recovered. Consider real values of the parameter $\lambda$, then the functions 
$t_{i j}(\lambda)$ are also real valued. Evaluating the real and imaginary parts of $a_{1} \pm \mathrm{i} b_{1}$ we may calculate the following four functions:

$$
\begin{aligned}
f_{1} & :=\left[(\alpha+1)(\delta-1)-\beta^{2}\right] t_{11} \\
+ & {\left[(\alpha-1)(\delta+1)-\beta^{2}\right] t_{22} ; } \\
f_{2} & :=\left[(\alpha+1)(\delta+1)-\beta^{2}\right] t_{21} \\
- & {\left[(\alpha-1)(\delta-1)-\beta^{2}\right] t_{12} ; } \\
f_{3} & :=[2 \beta \gamma \epsilon+\zeta(\alpha+1)(\delta-1) \\
& \left.-\epsilon^{2}(\alpha+1)-\gamma^{2}(\delta-1)-\beta^{2} \zeta\right] t_{11} \\
+ & {[2 \beta \gamma \epsilon+\zeta(\alpha-1)(\delta+1)} \\
& \left.-\epsilon^{2}(\alpha-1)-\gamma^{2}(\delta+1)-\beta^{2} \zeta\right] t_{22} ; \\
f_{4} & :=[2 \beta \gamma \epsilon+\zeta(\alpha+1)(\delta+1) \\
& \left.-\epsilon^{2}(\alpha+1)-\gamma^{2}(\delta+1)-\beta^{2} \zeta\right] t_{21} \\
& -[2 \beta \gamma \epsilon+\zeta(\alpha-1)(\delta-1) \\
& \left.-\epsilon^{2}(\alpha-1)-\gamma^{2}(\delta-1)-\beta^{2} \zeta\right] t_{12} .
\end{aligned}
$$

This system of linear equations can be divided into two $2 \times 2$ systems involving functions $\left(t_{11}, t_{22}\right)$ and $\left(t_{12}, t_{21}\right)$, but the corresponding determinants $D_{1,2}$ are identically equal to zero

$$
\begin{gathered}
D_{1}=2\left[2 \beta \gamma \epsilon(\delta-\alpha)+\epsilon^{2}\left(\alpha^{2}-1\right)\right. \\
\left.-\beta^{2} \epsilon^{2}-\gamma^{2}\left(\delta^{2}-1\right)+\beta^{2} \gamma^{2}\right]
\end{gathered}
$$

$$
\begin{aligned}
& =2\left[2 \beta \gamma \epsilon(\delta-\alpha)+\gamma^{2}\left(\epsilon^{2}+2 \beta^{2}\right)-\epsilon^{2}\left(2 \beta^{2}+\gamma^{2}\right)\right] \\
& =4\left[\gamma^{2} \beta^{2}-\epsilon^{2} \beta^{2}+\beta \gamma \epsilon \delta-\beta \gamma \epsilon \alpha\right] \\
& =4[\gamma \beta(\gamma \beta+\epsilon \delta)-\epsilon \beta(\epsilon \beta+\alpha \gamma)]=0 \\
D_{2} & =2\left[-2 \beta \gamma \epsilon(\alpha+\delta)+\epsilon^{2}\left(\alpha^{2}-1\right)\right. \\
& \left.+\epsilon^{2} \beta^{2}+\gamma^{2}\left(\delta^{2}-1\right)+\gamma^{2} \beta^{2}\right] \\
& =2\left[-2 \beta \gamma \epsilon(\alpha+\delta)+\epsilon^{2}\left(\alpha^{2}+\beta^{2}-1\right)\right. \\
& \left.+\gamma^{2}\left(\beta^{2} \delta^{2}-1\right)\right] \\
& =-4 \epsilon \gamma[\beta(\alpha+\delta) \epsilon \gamma]=0 .
\end{aligned}
$$

In the calculations above we used first that the row vectors in the matrix $S$ are orthogonal and then that they are normalized.

In fact it follows that the functions $t_{i j}$ in general cannot be reconstructed from the TW-function $M(\lambda, \Phi)$ known for all values of the magnetic flux $\Phi$. Assume first that all four coefficients appearing in the formulas for $f_{1,2}$ are different from zero

$$
(\alpha \pm 1)(\delta \pm 1)-\beta^{2} \neq 0 .
$$

We have in this case

$$
\left\{\begin{array}{l}
t_{11}=\frac{1}{(\alpha+1)(\delta-1)-\beta^{2}} f_{1}-\frac{(\alpha-1)(\delta+1)-\beta^{2}}{(\alpha+1)(\delta-1)-\beta^{2}} t_{22}, \\
t_{21}=\frac{1}{(\alpha+1)(\delta+1)-\beta^{2}} f_{1}+\frac{(\alpha-1)(\delta-1)-\beta^{2}}{(\alpha+1)(\delta+1)-\beta^{2}} t_{22} .
\end{array}\right.
$$

Taking into account that the transfer matrix has unit determinant we get

$$
\begin{aligned}
& -\frac{(\alpha-1)(\delta+1)-\beta^{2}}{(\alpha+1)(\delta-1)-\beta^{2}}\left(t_{22}-\frac{1}{2\left[(\alpha-1)(\delta+1)-\beta^{2}\right]} f_{1}\right)^{2}-\frac{(\alpha-1)(\delta-1)-\beta^{2}}{(\alpha+1)(\delta+1)-\beta^{2}}\left(t_{12}+\frac{1}{2\left[(\alpha-1)(\delta-1)-\beta^{2}\right]} f_{2}\right)^{2} \\
& \quad=1-\frac{1}{4\left[(\alpha-1)(\delta+1)-\beta^{2}\right]\left[(\alpha+1)(\delta-1)-\beta^{2}\right]} f_{1}^{2}-\frac{1}{4\left[(\alpha-1)(\delta-1)-\beta^{2}\right]\left[(\alpha+1)(\delta+1)-\beta^{2}\right]} f_{2}^{2} .
\end{aligned}
$$

The quotients on the left hand side are different from zero due to (4.16), therefore the analytic exponential type functions in the brackets are not uniquely determined by the function on the right hand side, even if one takes into account their standard asymptotic behavior. Consider any two such functions satisfying (4.17) and calculate the corresponding coefficients $t_{22}$ and $t_{12}$ in the transfer matrix. The two remaining coefficients $t_{11}$ and $t_{21}$ can be calculated from (4.15).

If just one of the coefficients in (4.16) is equal to zero, then one of the equations (4.15) allows to calculate one of the coefficients $t_{i j}$ directly, while the second equation determines the rest of the transfer matrix up to a certain sequence of signs. Assume without loss of generality that $(\alpha+1)(\delta-1)-\beta^{2} \neq 0$, then $t_{22}=f_{1} /\left[(\alpha-1)(\delta+1)-\beta^{2}\right]$. Consider the zeroes $k_{j}$ of $t_{22}: t_{22}\left(k_{j}\right)=0$, then taking into account that the transfer matrix has unit determinant we get

$$
t_{12}\left(k_{j}\right)=-1 / t_{21}\left(k_{j}\right),
$$

which leads to the following quadratic equation on $t_{12}\left(k_{j}\right)$

$$
\begin{aligned}
& {\left[(\alpha-1)(\delta-1)-\beta^{2}\right] t_{12}^{2}\left(k_{j}\right)+f_{2}\left(k_{j}^{2}\right) t_{12}\left(k_{j}\right)} \\
& \quad+\left[(\alpha+1)(\delta+1)-\beta^{2}\right]=0 .
\end{aligned}
$$

The corresponding discriminant

$$
f_{2}^{2}\left(k_{j}^{2}\right)-4\left[(\alpha-1)(\delta-1)-\beta^{2}\right]\left[(\alpha+1)(\delta+1)-\beta^{2}\right]
$$

is not equal to zero for all $j$, since otherwise all values $t_{12}\left(k_{j}\right)$ are equal and the function $t_{12}$ is a constant function. Therefore for each $j$ there exist two possible values of $t_{12}\left(k_{j}\right)$, which allows to find different exponential type functions $t_{12}$ with the necessary asymptotics. All such functions are parameterized by a sequence of signs similar to one appearing in the solution of the inverse problem for periodic Schrödingier operators [32, 33] (see also $[23])$. It follows that the transfer matrix and hence the potential on the loop is not uniquely determined.

\footnotetext{
$¥$ Note that the sequence is finite if the corresponding periodic Schrödinger operator has finite-band spectrum. 
The case where several coefficients in (4.15) are zero can be studied in a similar way.

We have shown that the potential on the loop in general is not determined by the magnetic flux dependent TW-function in the case of real Hermitian matrices $S$ parametrizing the matching conditions. It might be interesting to extend this analysis to the case of arbitrary unitary matrices and to analyze the cases where the potential is reconstructable.

We have shown that the potential on the loop in general is not determined by the magnetic flux dependent TW-function in the case of real Hermitian matrices $S$ parameterizing the matching conditions. It might be interesting to extend this analysis to the case of arbitrary unitary matrices and to analyze the cases where the potential is reconstructable.

\section{Acknowledgments}

The author was supported in part by Swedish Research Council grant \#50092501.

\section{References}

[1] S. Avdonin, P. Kurasov, Inverse Probl. Imaging 2, 1 (2008).

[2] S. Avdonin, P. Kurasov, M. Nowaczyk, Inverse Probl. Imaging 4, 579 (2010).

[3] S. Avdonin, P. Kurasov, Inverse problems for quantum trees III: recovering metric graph and complete solution of the problem.

[4] S. Avdonin, V. Mikhaylov, A. Rybkin, Commun. Math. Phys. 275, 791 (2005).

[5] M.I. Belishev, Inverse Problems 20, 647 (2004).

[6] M.I. Belishev, J. Math. Sci. (New York) 132, 11 (2006).

[7] M.I. Belishev, A.F. Vakulenko, J. Inverse Ill-Posed Probl. 14, 29 (2006).

[8] M.I. Belishev, Inverse Problems 23, R1 (2007).

[9] J. Boman, P. Kurasov, Adv. Appl. Math. 35, 58 (2005).

[10] B.M. Brown, R. Weikard, Proc. R. Soc. Lond. Ser. A Math. Phys. Eng. Sci. 461, 3231 (2005).

[11] L.D. Faddeev, Usp. Mat. Nauk 14, 57119 (1959).

[12] G. Freiling, V. Yurko, Appl. Anal. 86, 653 (2007).

[13] J. Garnett, E. Trubowitz, Comment. Math. Helv. 59, 258 (1984).

[14] J. Garnett, E. Trubowitz, Comment. Math. Helv. 62, 18 (1987).

[15] I.M. Gelfand, B.M. Levitan, Izv. Akad. Nauk SSSR, Ser. Mat. 15, 309360 (1951).
[16] N.I. Gerasimenko, B.S. Pavlov, Theoret. Math. Phys. 74, 230 (1988).

[17] N.I. Gerasimenko, Theoret. Math. Phys. 75, 460 (1988).

[18] B. Gutkin, U. Smilansky, J. Phys. A 34, 6061 (2001).

[19] P. Kurasov, M. Nowaczyk, J. Phys. A 38, 4901 (2005).

[20] P. Kurasov, F. Stenberg, J. Phys. A 35, 101 (2002).

[21] P. Kurasov, Ark. Matemat. 46, 95 (2008).

[22] P. Kurasov, J. Funct. Anal. 254, 934 (2008).

[23] P. Kurasov, Math. Proc. Cambridge Philos. Soc. 148, 331 (2010).

[24] P. Kurasov, Acta Phys. Pol. A 116, 765 (2009).

[25] P. Kurasov, "Can one distinguish quantum trees from the boundary?", accepted for publication in Proc. $A M S$.

[26] P. Kurasov, "How does magnetic field held to solve the inverse problem for quantum graphs?", in preparation.

[27] P. Kurasov, M. Enerbäck, "Aharonov-Bohm ring touching a quantum wire: how to model it and to solve the inverse problem", accepted for publication in Rep. Math. Phys.

[28] B.M. Levitan, M.G. Gasymov, Usp. Mat. Nauk 19, 3 (1964).

[29] Yu.I. Lyubarskiı̌, V.A. Marchenko, Funktsional. Anal. Prilozhen. 41, 58 (2007).

[30] H.P. McKean, E. Trubowitz, Commun. Pure Appl. Math. 29, 143 (1976).

[31] V.A. Marchenko, Usp. Mat. Nauk (N.S.) 12, 143145 (1957).

[32] V.A. Marchenko, I.V. Ostrovsky, Mat. Sb. (N.S.) 97, 540 (1975).

[33] V.A. Marchenko, I.V. Ostrovsky, Selecta Matemat. Soviet. 6, 101 (1987).

[34] V. Pivovarchik, Oper. Theory Adv. Appl. 124, 527 (2001).

[35] R. Rueckriemen, "Recovering quantum graphs from their Bloch spectrum", preprint arXiv:1101.6002v2, 10 Feb 2011.

[36] A. Rybkin, Inverse Probl. Imaging 3, 139 (2009).

[37] V. Yurko, Math. Notes 79, 572 (2006).

[38] V. Yurko, Inverse Problems 21, 1075 (2005).

[39] V. Yurko, Operators Matrices 2, 543 (2008).

[40] V. Yurko, Matem. Sbornik 200, 147 (2009); translation in Sbornik: Mathematics 200, 1403 (2009).

[41] V. Yurko, Inverse Problems 25, 105008 (2009).

[42] V. Yurko, Applied Math. Lett. 23, 875 (2010).

[43] V. Yurko, J. Inverse Ill-Posed Problems 18, 245 (2010). 